\title{
Surgery Research and Practice
}

ORIGINAL ARTICLE

\section{An Alternative Mesofemoral Shunt for Uncontrolled Variceal Bleeding}

\author{
Yi-Ju Wu ${ }^{1 *}$, Chih-Che Lin ${ }^{1}$, Yu-Hung Lin ${ }^{1}$, Chao-Long Chen ${ }^{1}$, Wayne Huang ${ }^{2}$ and Yu-Feng Kao ${ }^{3}$ \\ ${ }^{1}$ Department of Surgery, Kaohsiung Chang Gung Memorial Hospital, Chang Gung University College of \\ Medicine, Kaohsiung, Taiwan \\ ${ }^{2}$ Department of Surgery, Show Chwan Memorial Hospital, Chang-Hua, IRCAD, Taiwan \\ ${ }^{3}$ Department of Nursing Care, Kaohsiung Chang Gung Memorial Hospital, Chang Gung University \\ College of Medicine, Kaohsiung, Taiwan
}

*Corresponding author: Yi-Ju Wu, MD, Department of Surgery, Kaohsiung Chang Gung Memorial Hospital, Chang Gung University College of Medicine, No.123, Dapi Rd, Niaosong Dist, Kaohsiung City 833, Taiwan, Tel: +886-7-731-7123, Fax: $+886-7-731-8762$

\begin{abstract}
Background: Portal hypertension can lead to life-threatening variceal bleeding (VB). Transjugular intrahepatic portosystemic shunt (TIPSS) is the standard choice for uncontrolled VB. Unavailable for TIPSS, we design a mesofemoral shunt (MFS) as an alternative method for control of VB.

Methodology: From March 2011 to November 2014, eleven patients with VB due to liver cirrhosis were enrolled. The MFS was created by connecting from superior mesenteric vein below transverse colon, via subcutaneous tunnel, to right femoral vein with an 8-mm diameter polytetrafluoroethylene (PTFE) graft.

Results: The median follow-up was 13 months (range, 2 44 months). The MFS was successful in $11(100 \%)$ hemostasis. One patient died of surgical mortality and the other due to liver failure 6 months after MFS. The PTFE occlusion was documented by ultrasonography in $4(36 \%)$ patients. Two $(18 \%)$ developed recurrent bleeding, which was treated by surgical thrombectomy. Nine patients, including 2 bridged to LT, were alive.

Conclusion: The MFS is an effective and considerable shunt procedure to control VB in the situation of unavailable for TIPSS. The subcutaneous tunnel of MFS could also be managed as surgical thrombectomy.
\end{abstract}

\section{Keywords}

Mesofemoral shunt, Portal hypertension, Variceal bleeding

\section{Introduction}

Variceal bleeding (VB) caused by portal hypertension $(\mathrm{PHT})$ in the cirrhotic liver disease could be life threatening [1]. Multidisciplinary approaches are reported for controlling VB due to the PHT [2]. Initially, the non-operative methods, including the medication, endoscopy, and the Sengstaken-Blakemore (SB) tube are usually considered for controlling about $85 \%$ VB [2]. With failure of the non-operative methods, the portal system should be decompressed via a shunt between the portal and systemic venous circulation [2].

The transjugular intrahepatic portosystemic shunt (TIPS) has become the standard procedure of choice and is an ideal vehicle to serve as a "bridge" to liver transplantation (LT) [3]. But the requiring special kits or unavailable for the interventional radiologist with hightech procedure are concerned [3]. The surgery becomes the decisive step in this situation.

Traditionally, the shunted and non-shunted surgery could have the acceptable rates for control of VB. But all of the surgical methods need the approach of upper abdomen or dissection of hepatoduodenal ligaments, which could induce massive adhesion over the hepatic hilum. This adhesion involving the hepatic hilum or upper abdomen will have a negative impact on the following LT $[4,5]$.

Citation: Yi-Ju W, Chih-Che L, Yu-Hung L, Chao-Long C, Huang W, et al. (2018) An Alternative Mesofemoral Shunt for Uncontrolled Variceal Bleeding. Int J Surg Res Pract 5:086. doi.org/10.23937/23783397/1410086

Accepted: September 05, 2018 ; Published: September 07, 2018

Copyright: (c) 2018 Yi-Ju W, et al. This is an open-access article distributed under the terms of the Creative Commons Attribution License, which permits unrestricted use, distribution, and reproduction in any medium, provided the original author and source are credited. 


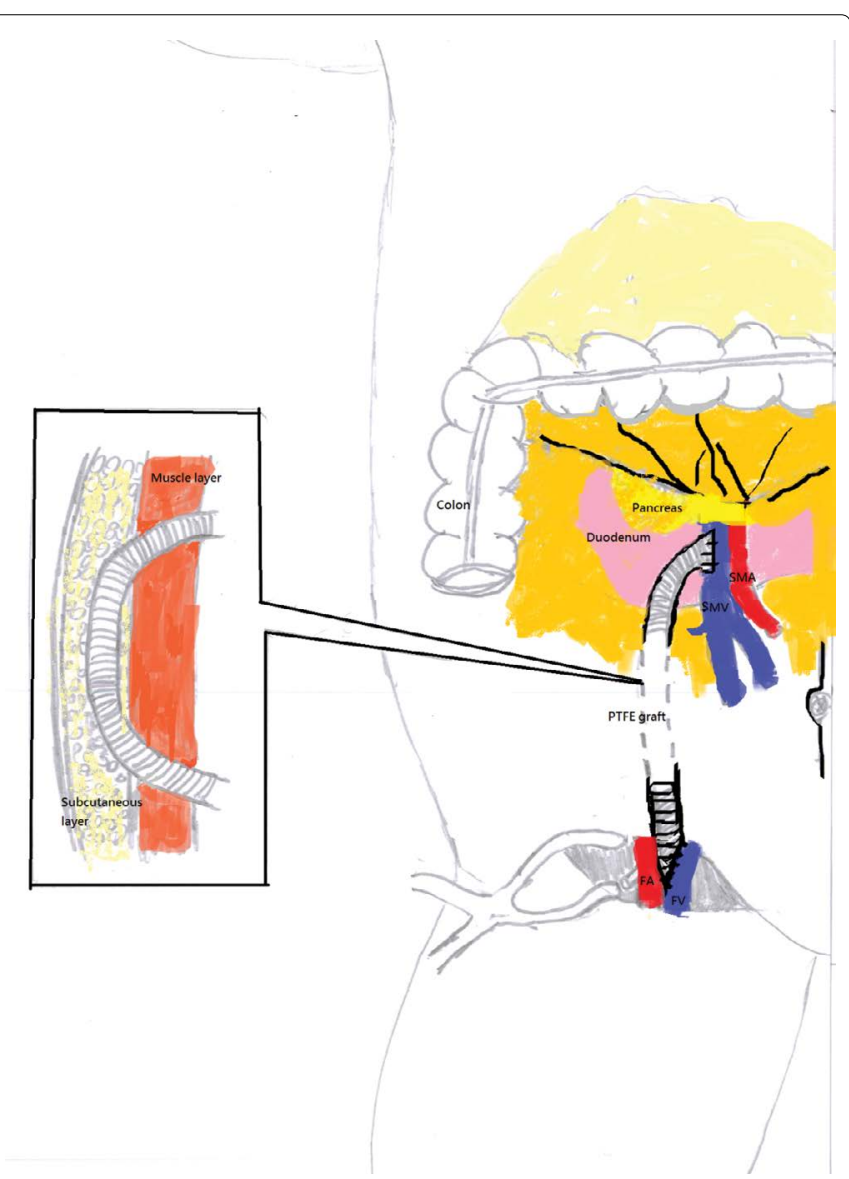

Figure 1: Mesofemoral shunt (MFS).

The MFS was created by connecting from superior mesenteric vein below transverse colon, via subcutaneous tunnel, to right femoral vein with an 8-mm-diameter PTFE graft.

SMV: Superior Mesenteric Vein; SMA: Superior Mesenteric Artery; FV: Femoral Vein; FA: Femoral Artery; PTFE: Polytetrafluroethylene.

In this paper we present the alternative procedure of mesofemoral shunt (MFS). The idea of MFS was originally from partial shunts such as portacaval or mesocaval shunts using small diameter of polytetrafluoroethylene (PTFE) H-graft [6,7]. Merging the concepts of partial shunt with decompression of PHT and avoidance of upper abdomen exploration are the prototype of our MFS. This alternative shunt could effectively control the VB for the patients who did not respond by medication, endoscopy, SB tube, or unavailable for TIPSS.

\section{Methodology}

\section{Patient selection}

From March 2011 to November 2014 after institutional review board approval, we enrolled the patients with VB due to cirrhotic liver disease. Patients were excluded if with hepatocellular carcinoma, previous operative history of abdomen, thrombosis of portal veins, or hepatic decompensation with imminent death. The clinical data and outcomes of eleven patients were reviewed. All had experienced bleeding from varices or portal gastropathy, and all had failed to medical therapy, endoscopic therapy, SB tube or unavailable for radiologic intervention.

\section{Surgical procedures}

General anesthesia with endotracheal intubation was employed. The patient was placed in the supine position. A peri-umbilical midline incision about 10 15 $\mathrm{cm}$ was used. With the transverse mesocolon retracted upward, a transverse incisionis made at the base of the transverse mesocolon. The superior mesenteric vein (SMV) was exposed between its first tributary and cephalic to almost the pancreas. The other inguinal incision about $5 \sim 8 \mathrm{~cm}$ was made just above the inguinal crease. Self-retracting retractors were positioned at both ends of the wound to enhance exposure. The femoral vein (FV) was dissected adequately for connection. Then a subcutaneous tunnel was created by the Kelly-Wick tunneler (Bard Peripheral Vascular, Inc, USA) to connect from the peri-umbilical incision to the right inguinal incision. The 8-mm-diameter externally ring-reinforced polytetrafluoroethylene (PTFE) vascular graft was placed in this tunnel. A Satinsky clamp was applied partially over the predetermined area on the superomedial surface of the SMV, a venotomy of appropriate size was made. The anastomosis with PTFE graft was made with non-absorbable suture material employing a continuous stitch. The graft was clamped at its opposite. The SMV clamp was released and the area inspected for adequate hemostasis. The graft was trimmed in oblique fashion to match the size of the venotomy of FV. The anastomosis again was made with a continuous stitch of non-absorbable suture material. No drains were used (Figure 1).

\section{Post-op care and follow up}

The adjustment of anticoagulation with wafarin was according to the international normalized ratio (INR): 1.5 2.0. Retrospectively reviewing the patients who were managed by the procedure in the past four years, the patients were regularly followed at median 13 months (range, 2 44 months) after surgery. Color-flow Doppler ultrasound was employed to check the patency of PTFE graft and documented for occlusion. If recurrent VB developed, surgical thrombectomy was performed via the PTFE graft in the subcutaneous tunnel through the groin incision. If it failed, we shifted to exploring the previous peri-umbilical incision and dissecting out the proximal end of PTFE graft below transverse colon. Surgical thrombectomy was performed in the same way through the previous peri-umbilical incision. Complications were recorded with the Clavien-Dindo classification [8].

\section{Results}

Eight males and three females underwent MFS at a median age of 44 years (range, 32-78 years). The causes of cirrhosis and PHT were alcohol abuse in 6, chronic hepatitis $C$ in 3 and chronic hepatitis $B$ in 2. There was Child-Turcotte-Pugh (CTP) class A in 1, class B in 7 and class $C$ in 3 . Eight patients had varices isolated to the 
Table 1: Demographics and clinical presentation of pre-MFS.

\begin{tabular}{|l|l|l|l|l|l|l|l|}
\hline No. & Age/Sex & Cause of cirrhosis & CTP & Bleeding & Ascites & Encephalopathy & MELD \\
\hline $\mathbf{1}$ & $56 / \mathrm{M}$ & HB & A & GV & none & none & 11 \\
\hline $\mathbf{2}$ & $40 / \mathrm{M}$ & HC & C & GV & mild & mild & 17 \\
\hline $\mathbf{3}$ & $32 / \mathrm{F}$ & Alcohol & B & EV+GV & mild & mild & 13 \\
\hline $\mathbf{4}$ & $49 / \mathrm{M}$ & HB & C & GV & mild & mild & 18 \\
\hline $\mathbf{5}$ & $43 / \mathrm{M}$ & Alcohol & B & EV+GV & mild & none & 14 \\
\hline $\mathbf{6}$ & 50/F & Alcohol & B & GV & mild & none & 11 \\
\hline $\mathbf{7}$ & $43 / \mathrm{M}$ & Alcohol & B & GV & mild & none & 12 \\
\hline $\mathbf{8}^{*}$ & 78/M & HC & B & GV & mild & none & 12 \\
\hline $\mathbf{9}$ & $43 / \mathrm{F}$ & Alcohol & B & GV & none & none & 11 \\
\hline $\mathbf{1 0}$ & 74/M & HC & B & GV & none & mild & 11 \\
\hline $\mathbf{1 1}$ & $44 / \mathrm{M}$ & Alcohol & & & & Severe & mild \\
\hline
\end{tabular}

with treated buccal carcinoma.

M: Male; F: Female; CTP: Child-Turcotte-Pugh classification; HB: Hepatitis B; HC: Hepatitis C; GV: Gastric Varices; EV: Esophagus Varices; MELD: Model for End-Stage Liver Disease.

Table 2: Surgical complications and mortality.

\begin{tabular}{|c|c|c|c|c|c|c|c|}
\hline No. & Complications & $\begin{array}{l}\text { Dindo-Clavien } \\
\text { classification }\end{array}$ & $\begin{array}{l}\text { Shunt occlusion } \\
\text { (m) }\end{array}$ & $\begin{array}{l}\text { Recurrent } \\
\text { bleeding (m) }\end{array}$ & LT (m) & $\begin{array}{l}\text { Follow-up } \\
\text { (m) }\end{array}$ & Mortality \\
\hline 1 & - & & 15 & - & & 44 & \\
\hline 2 & genital swelling & I & - & - & DDLT (3) & 43 & \\
\hline 3 & - & & 18 & 18 & & 30 & \\
\hline 4 & PSE & II & - & - & & 6 & + \\
\hline 5 & hemorrhoid & I & 4 & 4 & & 19 & \\
\hline 6 & - & & - & - & & 13 & \\
\hline 7 & PSE & II & - & - & $\operatorname{LDLT}(6)$ & 13 & \\
\hline 8 & - & & 12 & - & & 14 & \\
\hline 9 & liver failure & V & - & - & & 0.5 & + \\
\hline 10 & - & & - & - & & & \\
\hline 11 & PSE & II & - & - & & 2 & \\
\hline
\end{tabular}

m: Months after MFS; PSE: Portal-systemic encephalopathy; LTX: Liver transplantation; DDLT: Deceased donor liver transplantation; LDLT: Living donor liver transplantation.

stomach and three patients had both esophageal and gastric varices. Massive ascites combined with variceal bleeding happened to one of the Child's class $C$ patient. One 78-year-old male was with treated buccal carcinoma before. The demographics and clinical presentation of pre-MFS were described well in (Table 1).

\section{Surgical complications and mortality}

One Child's class C patient (9\%) died of liver failure 2 weeks later. According to the Clavien-Dindo classification, the surgical complications included 2 grade I, 2 grade II and 1 grade $V$. The 2 complications of grade I were hemorrhoid and genital swelling. These complications subsided spontaneously after physical therapy a few weeks later. The 2 complications of grade II were mild portal-systemic encephalopathy under control of ammonia-reducing medicine. The grade $V$ was aforementioned due to the liver failure. The other mortality happened to the patient with Child's class $C$ in 6 months later due to hepatic failure (Table 2).

\section{Outcomes}

The shunt procedure was initially successful at achieving hemostasis in 100\%, with a 91\% 30-day survival. The blood loss related with the procedure was no more than $300 \mathrm{ml}$ in each patient. The shunted occlu- sion by thrombosis was documented in the 4 patients (36\%). Two of the 4 patients with shunted occlusions developed recurrent bleeding (18\%). Recurrent bleeding happened to one patient 4 months after MFS and was treated by surgical thrombectomy via subcutaneous tunnel of PTFE graft through the groin incision of MFS. Recurrent bleeding happened to the other patient 18 months after MFS. Initially the groin incision was approached for surgical thrombectomy, but failed. Then we shifted to the previous peri-umbilical incision and dissected out the PTFE graft below transverse colon. Opened a hole over the PTFE graft and traced back to the proximal anastomosis. After surgical thrombectomy of proximal anastomosis and backflow of distal anastomosis, repair of the PTFE graft was finished.

Two patients were bridged to LT. One with Child's class $\mathrm{C}$ was fortunately bridged to deceased donor liver transplant 3 months after MFS. The other with Child's class B was bridged to living donor liver transplant from his daughter in 6 months later. Nine patients, including two bridged to LT, were alive after MFS (Table 2).

\section{Discussion}

In patients with poor liver function, management emphasis should be on LT. LT is the most effective treatment for PHT and end stage liver disease [2]. In patients 
Table 3: Comparison of MFS with other procedures.

\begin{tabular}{|l|l|l|l|l|l|l|l|l|}
\hline & & Type & $\begin{array}{l}\text { Bleeding } \\
\text { control }\end{array}$ & $\begin{array}{l}\text { Recurrent } \\
\text { bleeding }\end{array}$ & PSE & $\begin{array}{l}\text { Surgical } \\
\text { Mortality }\end{array}$ & $\begin{array}{l}\text { Related } \\
\text { blood } \\
\text { transfusion }\end{array}$ & $\begin{array}{l}\text { Upper } \\
\text { abdomen } \\
\text { adhesion }\end{array}$ \\
\hline Shunt & MFS & partial & $11(100 \%)$ & $2 / 11(18 \%)$ & $3 / 11(27 \%)$ & $1 / 11(9 \%)$ & none & none \\
\hline & TIPS (1,2) & non-selective & $90 \sim 100 \%$ & $10 \sim 26 \%$ & $20 \sim 30 \%$ & $15 \%$ & - & none \\
\hline & hMC (3)/hPC (4) & partial & $96 \sim 100 \%$ & $0 \sim 6 \%$ & $3 \sim 10.5 \%$ & $10.5 \sim 20 \%$ & - \\
\hline \\
\hline PCS (1,5) & non-selective & $97 \sim 100 \%$ & 0 & $15 \sim 40 \%$ & $13 \%$ & - & yes \\
\hline Non- & DSR (6) & selective & $95 \sim 99 \%$ & $5.4 \%$ & $11.7 \%$ & $6.40 \%$ & - & yes \\
\hline shunt & mS (7,8) & $\begin{array}{l}\text { devascularization/ } \\
\text { splenectomy }\end{array}$ & $100 \%$ & $<10 \%$ & $6.5 \%$ & $10 \sim 80 \%$ & $3 u(0 \sim 12)$ & yes \\
\hline
\end{tabular}

MFS: Mesofemoral shunt; TIPS: Transjugular intrahepatic portosystemic shunt; hMC: Small diameter of mesoacaval H-graft; hPC: Small diameter of portacaval H-graft; PCS: Portacaval shunt; DSRS: Distal splenorenal shunt; mS: Modified Sugiura procedure; PSE: Portal-systemic encephalopathy; -: Not provided.

with good liver function, management emphasis should be on control of VB [9]. In our study, the MFS achieved excellent results for uncontrolled VB. Instead of deeply approaching portal vein in the hepatoduodenal ligament and vena cava in the retroperitoneum, we approached SMV below transverse colon and FV over inguinal region. Both the SMV and FV were dissected out apparently. No related blood transfusion was required for the MFS. Venous pressure recorded from the common iliac vein or femoral vein can reflect that in the right atrium or central vein $[10,11]$. By diverting the portal pressure to the FV, the MFS could effectively lower the PHT and control the VB.

A TIPSS is the standard and life-rescuing procedure for VB due to PHT [3]. In patients with refractory bleeding who are at prohibitive risk for emergent surgery, TIPSS is successful at achieving hemostasis in $90 \%$, with a $63 \%$ 30-day survival [3]. Nevertheless, this data was achieved only by experienced interventional radiologist or specially trained physician. Special kits are also needed in well-equipped medical center [3]. Moreover, the reports of $20 \%$ liver failure due to decrease hepatic perfusion [7], 20\% 30\% worsening encephalopathy and shorter term of $50 \%$ shunt thrombosis or stenosis after TIPSS resulting in $26 \%$ recurrent bleeding are concerned $[3,7]$. Therefore, operative methods are necessary if the TIPSS procedures or other radiologic interventions have failed or unavailable.

Operative methods for the PHT of cirrhotic liver include the shunt and non-shunt procedures. According to the literature, the non-shunt operation (modified Sugiura/splenectomy) had a rate of recurrent bleeding less than $10 \%$ [12]. But the variable morbidity $(20 \sim 50 \%)$ and mortality $(10 \% \sim 80 \%)$ were reported $[12,13]$. Lots amount of blood transfusion are also required due to the massive dissection and friable vessels $[12,13]$. The shunted operation includes non-selective, selective and partial shunt. The non-selective shunted patients have excellent control of bleeding, but encephalopathy is severe in up to $40 \%$ of patients $[2,14]$. The distal splenorenal shunt (Warren's) is considered to be selective and less encephalopathy [2]. But time-requiring dissecting the retro-pancreatic splenic vein meticulous-
Iy and getting worsen ascites due to disruption of the retroperitoneal lymphatics are reported [2]. Moreover, multiple surgical morbidities were reported, including portal vein thrombosis, pancreatitis, pancreatic pseudocyst, and even pneumonia [15]. Partial shunt such as the portacaval or mesocaval shunt with small PTFEH-graft, which decompresses partially the portal pressure to the vena cava, is thought to maintain hepatopetal flow with improved survival and less encephalopathy. The MFS is also a partial shunt with small PTFE H-graft, which decompresses partially the portal pressure to the femoral vein. In our study, the MFS was very suitable for the Child's class A or B patients. The results of recurrent bleeding (18\%), shunt occlusion (36\%), or surgical mortality (9\%) were comparable to TIPSS (Table 3). The portal-systemic encephalopathy was controllable in the MFS patients. The transfemoral venography or angioplasty by interventional radiologist will be needed to detect and manage the occlusion of portacaval or mesocaval shunt. No reports were mentioned about the further correction if the radiologic intervention failed or unavailable $[6,7]$. Although mesocaval shunt leaves the hepatic hilum intact [6], it is necessary to approach upper abdomen and dissect deeply for vena cava. Overall, the reviewing literature of shunted and non-shunted operations, which need the approach of upper abdomen or dissection of hepatoduodenal ligaments, could result in the massive adhesions over hepatic hilum or upper abdomen. The surgery affecting the hepatic hilum or upper abdomen have a negative impact on orthotopic LT by increasing transfusion requirement; prolonging operative time, increasing ICU and total hospital duration of stay; and most importantly resulting in a higher perioperative mortality $[4,5]$. Essentially, we made the MFS below transvers colon in the lower abdomen and a subcutaneous tunnel to the femal vein. Even though the proximal graft caused massive adhesions to the gastrointestinal tract or transverse colon, we could easily dissected out the PTFE graft from the bowels and adhesions happened in the lower abdomen. The lower abdomen adhesions or surgery would not increase morbidity or mortality on orthotopic LT.

Some evidence suggests that the TIPSS should be applied in the patients with MELD (Model for End-stage 
Liver Disease) scores equal to or greater than 14 if LT is imminent; otherwise $\mathrm{H}$-graft of portacaval shunt should be used for patients of Child's class A or B or with MELD scores less than 14 if chances of later LT will be unlikely [7]. The question is that liver function is fluctuant and progressive with time. It may deteriorate a few months after shunted operation. Additionally, the patient with shunted operation may be informed or gets a chance for LT in few months later. At this time, the LT will encounter increased bleeding or other unpredictable complications due to the massive adhesions over upper abdomen or hepatic hilum. In our study, the two patients after MFS got chances for LT without anticipation before. One patient with Child's class $C$ got transplantation in 3 months later and the other with Child's class B in 6 months later. No adhesions over upper abdomen or hepatic hilum were encountered. Besides, the bleeding was decreased due to the decompression of PHT by MFS.

The occlusion rates $36 \%$ of MFS were higher than $10 \sim 12 \% \mathrm{H}$-graft of portacaval or mesocaval shunt $[6,7]$. The reasons of MFS for becoming thromobosed could be the longer the distance between the SMV and femoral vein, and a subcutaneous tunnel has greater mobility with abdominal wall and respiratory movement. However, it is very difficult for $\mathrm{H}$-graft of portacaval or mesocaval shunt to follow the patency by ultrasonography due to blockage of bowel's gas and manage the occlusion if unavailable for interventional radiologist. Conversely, in our MFS we could easily follow the patency of MFS in the subcutaneous layer by color Doppler ultrasonography. If shunted occlusions with recurrent VB occurred, the PTFE graft was dissected and managed as surgical thrombectomy in the subcutaneous layer of groin region or into abdominal cavity below transverse colon without UAE [16]. The maintained protocol of the PTFE graft is adjusted with warfarin to keep INR: 1.5 2 as the prevention of deep vein thrombosis. But some patients didn't took warfarin due to the prolong INR or hemorrhagic gastritis. In our study, the recurrent bleeding happened to two of 4 shunted occlusions, not all of them. According to the report of von Herby, et al. [17], they used the color Doppler ultrasonography to find spontaneous portasystemic shunts in 38\% of patients from a group of 109 cases with hepatic cirrhosis. We presumed that if the shunts were occluded few months after MFS, they would have the time to form spontaneous shunts by themselves. In this way, these spontaneous shunts could decompress PHT in replace of MFS.

Some limitations are worth noting in our study. First, the case number is small. However, we report these preliminary results and need more cases in the future. Second, the follow-up is not long enough. We may analyze the progressive change of liver function affected by the MFS. Third, we should collect and calculate the quantitative flow or pressure of portal vein before shunting. Try to define who should undergo which shunt or nonshunt procedures.

\section{Conclusion}

In conclusion, our preliminary report of MFS revealed the acceptable rate of patency, surgical complications and mortality. Additionally, the subcutaneous tunnel of MFS could be managed as surgical thrombectomy, even if the shunt occluded. It is also a good bridge to LT without increasing the surgical difficulty. The MFS is an effective and considerable shunt surgery to control VB in the situation of unavailable for TIPSS or other radiologic interventions.

\section{Acknowledgment}

We appreciated Mr. Yu-Feng Kao for his drawing work.

\section{Sources of Funding for Research}

Nil.

\section{Conflict of Interest}

Yi-Ju Wu and his co-authors have no conflict of interest.

\section{References}

1. Garcia-Tsao G, Bosch J, Groszmann RJ (2008) Portal hypertension and variceal bleeding--unresolved issues. Summary of an American Association for the study of liver diseases and European Association for the study of the liver single-topic conference. Hepatology 47: 1764-1772.

2. Wright AS, Rikkers LF (2005) Current management of portal hypertension. J Gastrointest Surg 9: 992-1005.

3. Boyer TD, Haskal ZJ, American Association for the Study of Liver Diseases (2005) The role of transjugular intrahepatic portosystemic shunt in the management of portal hypertension. Hepatology 41: 386-400.

4. Dell'Era A, Grande L, Barros-Schelotto P, Turnes J, Fuster J, et al. (2005) Impact of prior portosystemic shunt procedures on outcome of liver transplantation. Surgery 137: 620-625

5. Li C, Mi K, Wen TF, Yan LN, Li B, et al. (2012) Risk factors and outcomes of massive red blood cell transfusion following living donor liver transplantation. J Dig Dis 13: 161-167.

6. Descottes B, Lachachi F, Maisonnette F, Durand-Fontanier S, Abita T, et al. (2008) Long-term results of mesocaval shunts with polytetrafluoroethylene grafts. Int Surg 93: 268-273.

7. Rosemurgy AS, Bloomston M, Clark WC, Thometz DP, Zervos EE (2005) H-graft portacaval shunts versus TIPS: Ten-year follow-up of a randomized trial with comparison to predicted survivals. Ann Surg 241: 238-246.

8. Dindo D, Demartines N, Clavien PA (2004) Classification of surgical complications: A new proposal with evaluation in a cohort of 6336 patients and results of a survey. Ann Surg 240: 205-213.

9. Hermann RE, Henderson JM, Vogt DP, Mayes JT, Geisinger MA, et al. (1995) Fifty years of surgery for portal hypertension at the Cleveland Clinic Foundation. Lessons and prospects. Ann Surg 221: 459-468.

10. Pacheco Sda S, Machado MN, Amorim RC, Rol Jda L, Correa LC, et al. (2008) Central venous pressure in femoral catheter: Correlation with superior approach after heart surgery. Rev Bras Cir Cardiovasc 23: 488-493. 
11. Alzeer A, Arora S, Ansari Z, Fayed DF, Naguib M (1998) Central venous pressure from common iliac vein reflects right atrial pressure. Can J Anaesth 45: 798-801.

12. Selzner M, Tuttle-Newhall JE, Dahm F, Suhocki $P$, Clavien PA (2001) Current indication of a modified Sugiura procedure in the management of variceal bleeding. J Am Coll Surg 193: 166-173.

13. Voros D, Polydorou A, Polymeneas G, Vassiliou I, Melemeni A, et al. (2012) Long-term results with the modified Sugiura procedure for the management of variceal bleeding: Standing the test of time in the treatment of bleeding esophageal varices. World J Surg 36: 659-666.

14. Orloff MJ, Isenberg JI, Wheeler HO, Haynes KS, Horacio JB, et al. (2009) Portal-systemic encephalopathy in a ran- domized controlled trial of endoscopic sclerotherapy versus emergency portacaval shunt treatment of acutely bleeding esophageal varices in cirrhosis. Ann Surg 250: 598-610.

15. Elwood DR, Pomposelli JJ, Pomfret EA, Lewis WD, Jenkins $\mathrm{RL}$ (2006) Distal splenorenal shunt: Preferred treatment for recurrent variceal hemorrhage in the patient with well-compensated cirrhosis. Arch Surg 141: 385-388.

16. Augustinos P, Ouriel K (2004) Invasive approaches to treatment of venous thromboembolism. Circulation 110: 27-34.

17. von Herbay A, Frieling T, Haussinger D (2000) Color Doppler sonographic evaluation of spontaneous portosystemic shunts and inversion of portal venous flow in patients with cirrhosis. J Clin Ultrasound 28: 332-339. 